\title{
openheart Mortality and ventricular arrhythmia after acute myocarditis: a nationwide registry-based follow-up study
}

\author{
Kristian Hay Kragholm (10 , , ${ }^{1,2}$ Filip Lyng Lindgren, ${ }^{1}$ Tomas Zaremba (D) , ${ }^{1}$ \\ Phillip Freeman, ${ }^{1}$ Niels Holmark Andersen, ${ }^{1}$ Sam Riahi, ${ }^{1}$ Manan Pareek, ${ }^{3}$ \\ Lars Køber, ${ }^{4}$ Christian Torp-Pedersen, ${ }^{5}$ Peter Søgaard, ${ }^{1}$ Andreas Hagendorff, ${ }^{6}$ \\ Bhupendar Tayal ${ }^{1}$
}

To cite: Kragholm KH, Lindgren $\mathrm{FL}$, Zaremba T, et al. Mortality and ventricular arrhythmia after acute myocarditis: a nationwide registry-based follow-up study. Open Heart 2021;8:e001806. doi:10.1136/ openhrt-2021-001806

Received 4 August 2021 Accepted 1 October 2021

(D) Check for updates

(C) Author(s) (or their employer(s)) 2021. Re-use permitted under CC BY-NC. No commercial re-use. See rights and permissions. Published by BMJ.

${ }^{1}$ Department of Cardiology, Aalborg University Hospital, Aalborg, Denmark

${ }^{2}$ Unit of Clinical Biostatistics and Epidemiology, Aalborg University Hospital, Aalborg, Denmark ${ }^{3}$ Department of Cardiology, Nordsjællands Hospital, Hillerod, Denmark

${ }^{4}$ Department of Cardiology, Rigshospitalet, Copenhagen, Denmark

${ }^{5}$ Department of Cardiology, Nordsjaellands Hospital, Hillerod, Denmark

${ }^{6}$ Department of Cardiology, Leipzig University, Leipzig, Germany

Correspondence to Dr Kristian Hay Kragholm; kdks@rn.dk

\section{ABSTRACT}

Objective Incidence and severity of acute myocarditis vary significantly in previous reports and there is a lack of epidemiological studies on the short-term risks of mortality, heart failure and ventricular arrhythmias in patients with acute myocarditis. Therefore, study aims were to examine 90-day risks of mortality, heart failure (HF) and ventricular arrhythmias in patients with acute myocarditis in comparison to age-matched and sexmatched background population controls.

Methods In this nationwide register-based follow-up study of patients hospitalised with myocarditis between 2002 and 2018 in Denmark, 90-day risks of all-cause mortality, HF, ventricular arrhythmias (ventricular tachycardia, ventricular fibrillation (VF)), cardiac arrest and implantable cardioverter-defibrillator (ICD) implantation were compared with age-matched and sex-matched controls from the background population (1:5 matching). Absolute risks standardised to the age, sex and comorbidity distribution of the entire study population were derived from multivariable Cox regression. Results A total of 2523 patients hospitalised with myocarditis were included. Median age was 48 years (Q1Q3: $30-69$ ) and $67.7 \%$ were men. Comorbidity burden was more pronounced among patients with myocarditis relative to controls. Standardised 90-day all-cause mortality risk was $4.9 \%$ for patients with acute myocarditis versus $0.3 \%$ for controls $(p<0.001)$. Ninety-day standardised risks for other endpoints were $7.5 \%$ versus $0.1 \%$ for $\mathrm{HF}, 1.9 \%$ versus $<0.1 \%$ for VF/VF/arrest risk and $1.6 \%$ versus $<0.1 \%$ for ICD implantation (all $p<0.001$ ). Conclusions In this large nationwide register-based follow-up study, patients hospitalised with myocarditis had significantly higher 90-day risks of all-cause mortality, HF, ventricular arrhythmias, cardiac arrest and ICD implantation compared with background population controls.

\section{INTRODUCTION}

Myocarditis is an inflammation of the myocardium, primarily affecting young and otherwise healthy individuals, although it may also appear in patients with a history of cardiovascular disease. ${ }^{1}$ Its clinical presentation spans

\section{Key questions}

What is already known about this subject?

- Incidence and severity of acute myocarditis vary significantly in previous reports and there is a lack of epidemiological studies on the short-term risks of mortality, heart failure and ventricular arrhythmias in patients with acute myocarditis.

What does this study add?

- In this large nationwide register-based follow-up study of 2523 patients hospitalised with myocarditis, 90-day risks of all-cause mortality, heart failure, ventricular arrhythmias, cardiac arrest and implantable cardioverter-defibrillator implantation were significantly higher when compared with background population controls.

How might this impact on clinical practice?

- These findings suggest that patients with acute myocarditis may benefit from careful diagnostic work-up including cardiac monitoring in the early phase after diagnosis. Further studies focusing on risk stratification for these patients are warranted.

from almost asymptomatic patients, with mild palpitations or chest pain, to cardiovascular collapse and death. ${ }^{2}$ In its mild form, myocarditis often resolves without any treatment. However, in its more fulminant form, it may account for up to $42 \%$ of the events of sudden cardiac death among young, often athletic, individuals. ${ }^{3-5}$ Patients who survive have an increased risk of arrhythmias, dilated cardiomyopathy and death compared with the general population. ${ }^{6-9}$ Autopsy reports estimate an incidence of acute myocarditis ranging from $0.1 \%$ to up to $10 \% .^{310-12}$ Given a lack of epidemiological studies, and because estimates of incidence and severity of acute myocarditis vary significantly, we investigated short-term (90-day) risks of mortality, heart failure (HF) and ventricular arrhythmias (ventricular tachycardia (VT), 
ventricular fibrillation (VF), cardiac arrest and implantable cardioverter-defibrillator (ICD) implantation procedures) in patients hospitalised with myocarditis in Denmark between 2002 and 2018 and compared with age-matched and sex-matched population controls using a nationwide registry-based follow-up design.

\section{METHODS}

\section{Data sources and study population}

This nationwide registry-based follow-up study is based on The Danish Civil Registration System containing all registered Danish citizens, their unique personal identification numbers, sex, date of birth and date of death. ${ }^{13}$ This registry can be used to link data to other nationwide administrative databases. The Danish National Patient Register was used to include all patients between 2002 and 2018 with a first-time hospitalisation with a myocarditis diagnosis, either as a primary or a secondary diagnosis with a primary diagnosis of $\mathrm{HF}, \mathrm{VT} / \mathrm{VF} /$ arrest, ICD implantation or atrial fibrillation or flutter $(\mathrm{AF}) .{ }^{14}$ These secondary diagnoses were chosen as the clinical presentation of acute myocarditis may involve $\mathrm{HF}, \mathrm{VT} / \mathrm{VF}$ /arrest, $\mathrm{AF}$ and/or lead to ICD implantation. The acute myocarditis population was age-matched and sex-matched to population controls, without a diagnosis of prior acute myocarditis, in a 1:5 ratio.

\section{Diagnostic work-up for acute myocarditis}

The Danish National Patient Register was used to obtain data on pre-existing medical conditions, medical examinations and hospital and outpatient contacts including relevant diagnoses, procedures and surgical codes. The diagnostic work-up for acute myocarditis includes echocardiography and although we did have data on whether coronary CT angiography (CCTA), coronary angiogram (CAG), positron emission tomography CT (PET-CT), endomyocardial biopsy (EMB) and cardiac MRI (CMR) additionally were performed, results of all these investigations including echocardiography, as well as ECG and cardiac biomarkers (troponins) were not available from the registry. The role of CCTA and CAG in the diagnostic work-up was to rule out ischaemic heart disease. Diagnostic work-up was assessed in a 30-day time span both prior to and 30 days after the diagnosis of acute myocarditis. Furthermore, data on mortality at follow-up were collected in relation to each diagnostic work-up. As CMR was first introduced as a diagnostic tool in Denmark in 2007, the number of performed CMRs relative to other examinations was expected to be low.

\section{Other study variables}

In addition to patient age and sex, prior cardiac procedures including percutaneous coronary intervention (PCI), coronary artery bypass grafting (CABG) and ICD implantations as well as the following comorbid conditions were assessed within the last 5 years prior to the acute myocarditis index date using the Danish National Patient Registry: hypertension, diabetes, chronic obstructive pulmonary disease (COPD), prior myocardial infarction (MI), prior ischaemic heart disease (IHD), AF, stroke, peripheral artery disease (PAD), prior HF, prior $\mathrm{VT} / \mathrm{VF}$, cardiac arrest and chronic kidney disease (CKD). Hypertension, diabetes and COPD were defined as either a relevant diagnosis code or relevant and specific prescription medication within the last 180 days prior to the acute myocarditis index date using data from the Danish Prescription Registry. ${ }^{15}$ As done previously, the prescription medication definition of hypertension was redemption of at least two antihypertensive drugs in two consecutive quarters prior to study inclusion. ${ }^{16}$

\section{Outcomes}

The primary outcome was 90-day all-cause mortality. Secondary outcomes were 90-day presumed cardiovascular cause of death and 90-day risks of HF, a composite of VT or VF or cardiac arrest (VT/VF/arrest) and ICD implantation. Cardiac arrest was defined as a diagnosis made in the hospital and registered in The Danish National Patient Register.

\section{Statistical analysis}

Continuous data are presented as median and first to third quartiles (Q1-Q3) and categorical data as counts and percentages. Mann-Whitney and Pearson's $\chi^{2}$ tests were performed to report crude between-group differences. Kaplan-Meier and Aalen-Johansen estimators were calculated to present crude absolute risks of mortality and outcomes of HF, a composite of VT or VF or cardiac arrest (VT/VF/arrest) and ICD implantation. Adjusted HRs and absolute risks for patients with acute myocarditis versus population controls standardised to the age, sex and comorbidity distribution of the entire study population were derived from multivariable Cox regression. ${ }^{17}$ Standardisation ensures that when patients with myocarditis are compared with population controls, all covariates including age, sex and comorbidities are held equal between the two comparison groups. The following covariates were included in the models: age, sex, hypertension, diabetes, COPD, prior MI, prior IHD, PCI, CABG, stroke, $\mathrm{PAD}, \mathrm{AF}, \mathrm{HF}$, prior VT/VF/arrest and CKD. Sensitivity analyses were performed on patients $<60$ years since a prior study showed that the diagnostic accuracy of acute myocarditis from the Danish National Patient Registry was high in this age group. ${ }^{18}$ Since IHD is a potential differential diagnosis to myocarditis, a sensitivity analysis in which MI or revascularisation events were treated as competing risks were additionally performed to exclude that outcome events were driven by IHD. Two-sided $p$ values $<0.05$ were considered statistically significant. Data management and analyses were performed using SAS, V.9.4 and R, V.3.6.1. ${ }^{19}$

\section{Patient consent and ethical approval}

According to Danish legislation, ethical committee approval or patient consent is not required for the conduct of register-based studies. In compliance with the 
Table 1 Characteristics for patients with acute myocarditis versus age-matched and sex-matched population controls

\begin{tabular}{|c|c|c|c|}
\hline & $\begin{array}{l}\text { Patients } \\
\text { with acute } \\
\text { myocarditis }\end{array}$ & $\begin{array}{l}\text { Population } \\
\text { controls }\end{array}$ & \\
\hline Variable & $(n=2523)$ & $(n=12615)$ & $P$ value \\
\hline Age, median (Q1-Q3) & $49(30-69)$ & $48(30-69)$ & 0.98 \\
\hline Male sex, n (\%) & $1709(67.7)$ & $8545(67.7)$ & $>0.99$ \\
\hline Hypertension, n (\%) & $804(31.9)$ & 2019 (16.0) & $<0.001$ \\
\hline Diabetes, n (\%) & $194(7.7)$ & $301(2.4)$ & $<0.001$ \\
\hline COPD, n (\%) & $165(6.5)$ & $223(1.8)$ & $<0.001$ \\
\hline Prior MI, n (\%) & $184(7.3)$ & $145(1.1)$ & $<0.001$ \\
\hline Prior IHD, n (\%) & $396(15.7)$ & $444(3.5)$ & $<0.001$ \\
\hline Prior PCl, n (\%) & $58(2.3)$ & $93(0.7)$ & $<0.001$ \\
\hline Prior CABG, n (\%) & $23(0.9)$ & $32(0.3)$ & $<0.001$ \\
\hline AF, n (\%) & $373(14.8)$ & $318(2.5)$ & $<0.001$ \\
\hline Stroke, n (\%) & $114(4.5)$ & $233(1.8)$ & $<0.001$ \\
\hline PAD, n (\%) & $80(3.2)$ & $123(1.0)$ & $<0.001$ \\
\hline Prior AV block, n (\%) & $35(1.4)$ & $37(0.3)$ & $<0.001$ \\
\hline Prior HF, n (\%) & $324(12.8)$ & $165(1.3)$ & $<0.001$ \\
\hline Prior VT/NF/arrest, n (\%) & $45(1.8)$ & $18(0.1)$ & $<0.001$ \\
\hline Prior ICD, n (\%) & $40(1.6)$ & $10(0.1)$ & $<0.001$ \\
\hline CKD, n (\%) & $72(2.9)$ & $86(0.7)$ & $<0.001$ \\
\hline
\end{tabular}

Q1-Q3: first-third quartile (25\%-75\%).

$\mathrm{AF}$, atrial fibrillation or flutter; $\mathrm{AV}$, atrioventricular; $\mathrm{CABG}$, coronary artery bypass grafting; CKD, chronic kidney disease; COPD, chronic obstructive pulmonary disease; HF, heart failure; ICD, implantable cardioverter-defibrillator; IHD, ischaemic heart disease; MI, myocardial infarction; PAD, peripheral artery disease; $\mathrm{PCl}$, percutaneous coronary intervention; ; VF, ventricular fibrillation; VT, ventricular tachycardia.

General Data Protection Regulation, the data responsible institute in the Capital Region of Denmark has approved the use of the data sources for research purposes (approval number P-2019-404).

\section{RESULTS}

\section{Patients, characteristics and diagnostic work-up}

A total of 15138 subjects were included, 2523 in the acute myocarditis group and 12615 in the control group. Of the 2523 patients hospitalised with myocarditis, 2104 had a primary diagnosis of myocarditis and 419 had a diagnosis of myocarditis secondary to $\mathrm{HF}, \mathrm{VT} / \mathrm{VF}$ /arrest, ICD device implantation or AF. Median age of patients with acute myocarditis was 48 years (Q1-Q3: 30-69 years) and approximately two-thirds were men $(67.7 \%)$. The distribution of comorbidities in patients with acute myocarditis and population controls is listed in table 1. Diagnostic studies performed for patients with acute myocarditis are shown in table 2. Approximately $45 \%$ and $5 \%$ underwent CAG and CCTA, respectively, to rule out IHD as part of the diagnostic work-up. During 2002-2007, 33 of 796 (4.2\%) patients underwent either CMR $(n=3)$ or $\operatorname{EMB}(n=30)$,
Table 2 Diagnostic work-up, advanced in-hospital treatment and selected post-discharge medications for the 2523 patients with acute myocarditis

\begin{tabular}{lc}
\hline Variable & $\mathbf{n}(\%)$ \\
\hline Diagnostic modality & $144(5.7)$ \\
\hline CCTA & $1137(45.1)$ \\
\hline CAG & $9(0.4)$ \\
\hline PET-CT & $214(8.5)$ \\
\hline CMR & $98(3.9)$ \\
\hline EMB & $285(11.3)$ \\
\hline PET-CT or CMR or EMB & $283(11.2)$ \\
\hline CMR or EMB & \\
\hline In-hospital advanced care & $182(7.2)$ \\
\hline ICU admission & $112(4.4)$ \\
\hline Inotropic agent use & $16(0.6)$ \\
\hline ECMO & $9(0.4)$ \\
\hline IABP & $4(0.2)$ \\
\hline T-LVAD & \\
\hline Post-discharge medication & $455(18.0)$ \\
\hline NSAID & $45(1.8)$ \\
\hline Colchicin & $133(5.3)$ \\
\hline Steroid & $658(26.1)$ \\
\hline ACEi/ARB & $761(30.2)$ \\
\hline Beta-blocker & \\
\hline ACE
\end{tabular}

$\mathrm{ACEi}$, angiotensin-converting enzyme inhibitor; ARB, angiotensinII receptor blocker; CAG, coronary angiogram; CCTA, coronary CT angiography; CMR, cardiac magnetic resonance; ECMO, extracorporeal membrane oxygenation; EMB, endomyocardial biopsy; IABP, intra-aortic balloon pump; ICU, intensive care unit; NSAID, non-steroidal anti-inflammatory drug; PET-CT, positron emission tomography CT; T-LVAD, temporary left ventricular assist device.

whereas 250 of 1727 (14.5\%) underwent EMB ( $\mathrm{n}=68)$ and/or CMR ( $\mathrm{n}=211)$ during 2008-2018. EMB use was stable over time, with 30 procedures recorded during 2002-2007 (3.8\%) versus $68(3.9 \%)$ during 2008-2018. CMR was performed in $3(0.4 \%)$ patients during 2002 2007 and in $211(12.2 \%)$ patients during 2008-2018. In total, only nine patients $(0.4 \%)$ underwent PET-CT.

\section{Outcomes for patients with acute myocarditis versus population controls}

Relative to population controls, patients with acute myocarditis had significantly higher crude incidences of all-cause mortality, HF, VT or VF or cardiac arrest and ICD implantation within 90 days of follow-up (figure 1). These differences remained significant when standardising the absolute risks to the age, sex and comorbidity distribution of the entire study population: 90-day allcause mortality risks of $4.9 \%$ versus $0.3 \%, 90$-day HF risks of $7.5 \%$ versus $0.1 \%, 90$-day $\mathrm{VF} / \mathrm{VF} /$ arrest risks of $1.9 \%$ versus $<0.1 \%$ and 90 -day ICD implantation risks of $1.6 \%$ 
A 90-day mortality

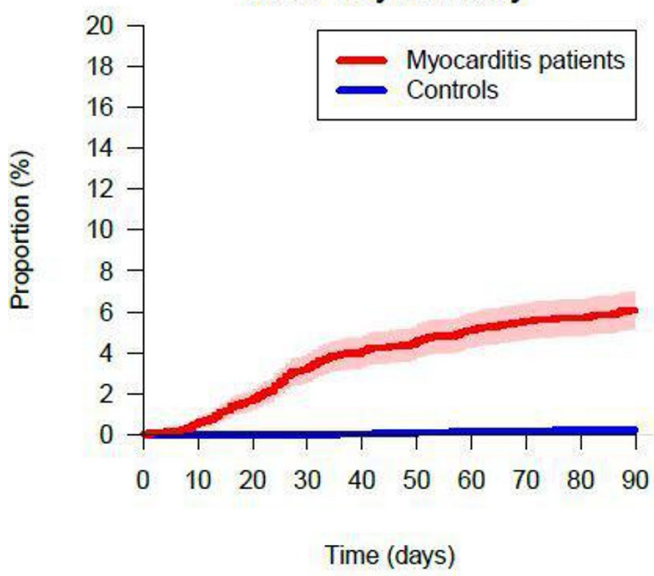

C 90-day VT/VF/arrest risk

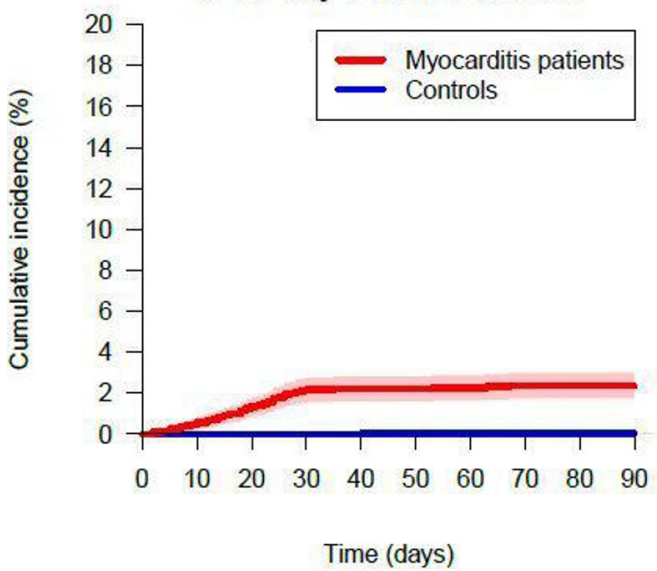

B 90-day HF risk

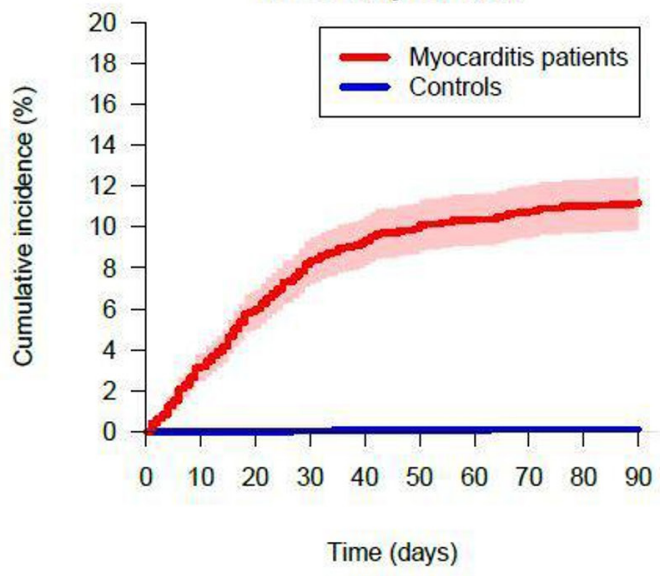

D 90-day ICD implantation risk

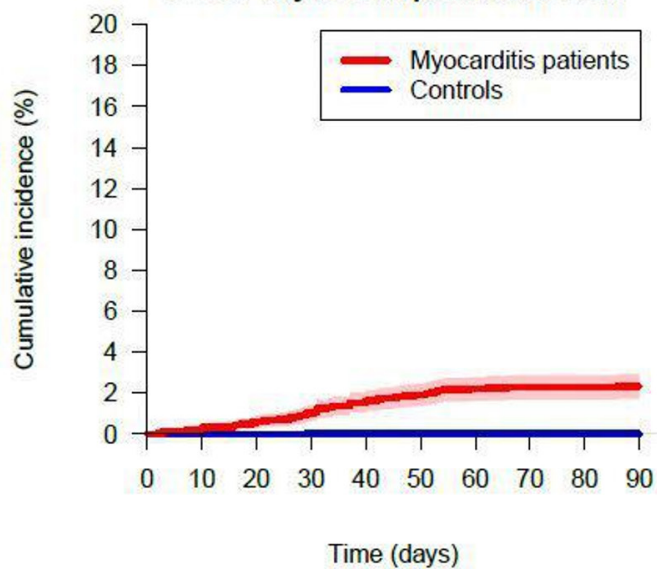

Figure 1 Crude 90-day outcomes of all-cause mortality, HF, ventricular arrhythmias and ICD implantation. HF, heart failure; ICD, implantable cardioverter-defibrillator; VF, ventricular fibrillation; VT, ventricular tachycardia.

versus $<0.1 \%$ (figure 2). The corresponding HRs are shown in figure 3 .

\section{Ventricular arrhythmic events among patients with myocarditis}

Among the 2523 patients with myocarditis, 54 (2.1\%) had an arrhythmic event of either VT/VF or cardiac arrest within 90 days of follow-up. Of these 54 cases, 12 (22.2\%) died within 90 days of follow-up and $23(42.6 \%)$ had an ICD implanted within 90 days of follow-up. Among 57 $(2.3 \%)$ of the patients with myocarditis who had an ICD implanted within 90 days, the 23 patients with a VT/VF/ arrest event represented $38.3 \%$ of all who had an ICD implanted.

\section{Sensitivity analyses of patients below $\mathbf{6 0}$ years of age}

A total of 1445 patients with acute myocarditis and 7225 controls formed the basis for the sensitivity analyses of patients $<60$ years of age. Although overall event rates were lower compared with the complete study population, the risks remained significantly higher for patients with acute myocarditis relative to controls, with 90-day mortality risks of $1.1 \%$ (95\% CI $0.5 \%$ to $1.7 \%$ ) versus $<0.1 \%(\mathrm{p}<0.001), 90$-day HF risks of $5.8 \%$ (95\% CI $4.6 \%$ to $7.1 \%)$ versus $<0.1 \%(\mathrm{p}<0.001), 90$-day $\mathrm{VT} / \mathrm{VF} /$ arrest risks of $1.5 \%$ (95\% CI $0.8 \%$ to $2.2 \%$ ) versus $<0.1 \%$ $(\mathrm{p}<0.001)$ and 90-day ICD implantation risks of $1.7 \%$ (95\% CI $1.0 \%$ to $2.4 \%)$ versus $<0.1 \%(p<0.001)$.

\section{Other sensitivity analyses}

All-cause mortality in relation to whether specific diagnostic studies were performed is shown in table 3. Patients who underwent EMB had significantly higher mortality, potentially indicating a more severe disease course of the myocarditis condition. Standardised absolute risks of the study outcomes among patients with myocarditis diagnosed before versus after increasing availability and use of CMR (years 2002-2007 vs 2008-2018) are shown in table 4. The standardised absolute 90-day VT/VF/ arrest risk was significantly lower in 2008-2018 versus in 2002-2007, with $1.6 \%$ versus $3.3 \%$. Results from analyses of the 90-day HF risk in patients with acute myocarditis on exclusion of patients with prior HF $(6.8 \%$ for patients with acute myocarditis versus $<0.1 \%$ for controls), as well as results from analyses of the 90-day risk of VT/VF/ 
arrest in patients with acute myocarditis without prior $\mathrm{VT} / \mathrm{VF} /$ arrest $(1.8 \%$ for patients with acute myocarditis versus $<0.1 \%$ for controls) were comparable to the main study findings. Outcomes when MI or revascularisation events during the 90-day follow-up period were considered competing risks were comparable to the overall results and can be found in figure 4 .

\section{DISCUSSION}

This Danish nationwide registry-based study showed that 90-day incidences of all-cause mortality, HF, ventricular arrhythmias, cardiac arrest and ICD implantation were significantly higher among patients with acute myocarditis compared with controls. This may entail a more careful diagnostic work-up and monitoring strategy in the early phase after diagnosis of acute myocarditis to identify patients at high risk for incident cardiovascular events.

There are a very limited numbers of study with large data who have investigated the incidence of adverse cardiovascular events after acute myocarditis. There is a wide variability in the incidences of adverse cardiovascular outcomes among patients with acute myocarditis. As an example, reported incidences of dilated cardiomyopathy among patients with histologically positive acute myocarditis have ranged between $14 \%$ and $50 \% .^{20}$ In a recent series of 174 patients with EMB (and thus Dallas criteria $^{21}$ ) positive myocarditis, $17 \%$ suffered death or heart transplantation within the first 2 years. ${ }^{22}$ Moreover, a large Spanish national health service registry study $(\mathrm{N}=14147)$ reported a 3\% in-hospital mortality among patients admitted with acute myocarditis between 2003 and $2015 .^{23}$ This appears to be fairly compatible with our results considering that our study included follow-up data until 3 months post diagnosis. Part of the variability in outcomes is likely related to the heterogeneity of the

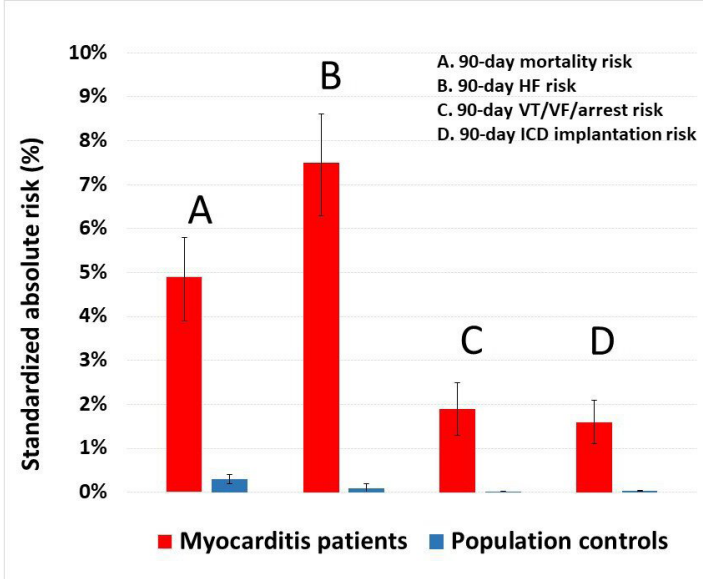

Figure 2 Standardised 90-day absolute risks of all-cause mortality, HF, ventricular arrhythmias and ICD implantation. The reported absolute risks for patients with acute myocarditis versus population controls standardised to the age, sex and comorbidity distribution of all patients were derived from multivariable Cox regression. The following covariates were included in the models: patient age, sex, hypertension, diabetes, chronic obstructive pulmonary disease, prior myocardial infarction, prior ischaemic heart disease, percutaneous cardiac intervention, coronary artery bypass grafting, stroke, peripheral artery disease, atrial fibrillation or flutter, HF, prior ventricular VT/NF/arrest and chronic kidney disease. HF, heart failure; ICD, implantable cardioverter-defibrillator; VF, ventricular fibrillation; VT, ventricular tachycardia.

myocarditis condition, with multiple underlying causes with distinct prognosis, warranting further investigation.

Complementing prior research, ${ }^{3-5} 824$ our study also suggests that acute myocarditis is a severe disease associated with a significantly increased short-term risk of death. Even though patients with acute myocarditis had more preexisting comorbidities compared with the age-matched

\begin{tabular}{lllll|} 
Outcome & HR Low 95\% & High 95\% & P-value \\
$\begin{array}{l}\text { A 90-day mortality } \\
\text { Myocarditis patients vs controls }\end{array}$ & 22.12 & 14.44 & 33.88 & $<0.001$ \\
$\begin{array}{l}\text { B 90-day HF risk } \\
\text { Myocarditis patients vs controls }\end{array}$ & 75.29 & 42.54 & 133.23 & $<0.001$ \\
$\begin{array}{l}\text { C 90-day VT/VFlarrest risk } \\
\text { Myocarditis patients vs controls }\end{array}$ & 78.80 & 24.19 & 256.65 & $<0.001$ \\
$\begin{array}{l}\text { D 90-day ICD implantation } \\
\text { Myocarditis patients vs controls }\end{array}$ & 65.56 & 20.10 & 213.90 & $<0.001$
\end{tabular}

Figure 3 Adjusted HRs of 90-day all-cause mortality, HF, ventricular arrhythmias and ICD implantation for patients with acute myocarditis vs age-matched and sex-matched population controls. The multivariable Cox regression models included the following covariates: patient age, sex, hypertension, diabetes, chronic obstructive pulmonary disease, prior myocardial infarction, prior ischaemic heart disease, percutaneous cardiac intervention, coronary artery bypass grafting, stroke, peripheral artery disease, atrial fibrillation or flutter, HF, prior ventricular VT/VF/arrest and chronic kidney disease. HF, heart failure; ICD, implantable cardioverter-defibrillator; VF, ventricular fibrillation; VT, ventricular tachycardia. 
Table 3 Standardised 90-day all-cause mortality risks in relation to diagnostic work-up

\begin{tabular}{|c|c|c|c|}
\hline \multirow[b]{2}{*}{ Diagnostic modality } & Diagnostic work-up & Diagnostic work-up & \multirow[b]{2}{*}{$P$ value } \\
\hline & Performed & Not performed & \\
\hline СCTA, risk (95\% Cl) & $3.9 \%(0.4 \%$ to $7.3 \%)$ & $6.2 \%(5.2 \%$ to $7.1 \%)$ & 0.21 \\
\hline CAG, risk (95\% Cl) & $4.3 \%$ (3.0\% to $5.6 \%)$ & $7.4 \%(5.9 \%$ to $8.8 \%)$ & 0.004 \\
\hline PET-CT, risk (95\% Cl) & $0.0 \%(0.0 \%$ to $0.0 \%)$ & $6.1 \%(5.1 \%$ to $7.0 \%)$ & $<0.001$ \\
\hline CMR, risk (95\% Cl) & $6.3 \%(1.4 \%$ to $11.3 \%)$ & $6.1 \%(5.1 \%$ to $7.0 \%)$ & 0.92 \\
\hline EMB, risk (95\% Cl) & $15.1 \%(8.2 \%$ to $22.1 \%)$ & $5.8 \%(4.9 \%$ to $6.7 \%)$ & 0.009 \\
\hline PET-CT or CMR or EMB, risk (95\% Cl) & $9.4 \%$ (4.9\% to $13.9 \%)$ & $5.9 \%(4.9 \%$ to $6.8 \%)$ & 0.13 \\
\hline CMR or EMB, risk $(95 \% \mathrm{Cl})$ & $9.5 \%(5.0 \%$ to $14.1 \%)$ & $5.9 \%(4.9 \%$ to $6.8 \%)$ & 0.13 \\
\hline
\end{tabular}

Number of patients who had the specific diagnostic work-up modalities performed can be found in table 1, and the comparator consists of the acute myocarditis population $(n=2523)$ minus the number of the patients who had the diagnostic modality performed. The reported absolute risks for patients with acute myocarditis versus population controls standardised to the age, sex and comorbidity distribution of all patients were derived from multivariable Cox regression. The following covariates were included in the models: patient age, sex, hypertension, diabetes, chronic obstructive pulmonary disease, prior myocardial infarction, prior ischaemic heart disease, percutaneous cardiac intervention, coronary artery bypass grafting, stroke, peripheral artery disease, atrial fibrillation or flutter, heart failure, prior ventricular tachycardia/ventricular fibrillation/ arrest and chronic kidney disease.

CAG, coronary angiogram; CCTA, coronary CT angiography; CMR, cardiac magnetic resonance; EMB, endomyocardial biopsy; PET-CT, positron emission tomography CT.

and sex-matched population controls in our study, the acute myocarditis population still had a significantly elevated mortality risk when standardising to the age, sex and comorbidity distribution of all individuals included. In this analysis, the 90 -day mortality risk was $4.9 \%$ for patients with acute myocarditis versus $0.3 \%$ for the age-matched and sex-matched population controls. This emphasises the importance of rapid diagnosis and targeted management to potentially improve survival. In addition to the increased mortality risk, risks of ventricular arrhythmias, cardiac arrest and ICD implantation were also substantially higher among patients with acute myocarditis relative to the population controls. Accordingly, in-hospital and additionally post-discharge cardiac monitoring of patients with acute myocarditis may aid in early arrhythmia detection to potentially prevent fatal outcomes. As for the increased short-term risk of HF, this may have clinical implications in the sense that early detection of left ventricular dysfunction would allow for earlier initiation of guideline-directed medical and device therapy.

EMB use was stable just below $4 \%$ throughout the study period. Although this seems low, use of EMB is limited in routine clinical practice and generally reserved for the most severe cases. This is also supported by our results, as we observed significantly higher event rates in patients who underwent EMB relative to those who did not. Several explanations may be provided for the limited use of EMB. First, acute myocarditis is usually associated with focal inflammation and may involve a region inaccessible by biopsy. ${ }^{25}$ Second, there seems to be poor agreement between observers. ${ }^{26}$ Finally, timely performance of EMB is important as the degree of inflammation may decrease over time, but this may not always be possible due to lack of facilities or adequate clinical expertise.

Table 4 Standardised 90-day risks in relation to periods 2008-2018 versus 2002-2007 among the 2523 patients with acute myocarditis

\begin{tabular}{|c|c|c|c|}
\hline & 2008-2018 & 2002-2007 & \\
\hline Outcome measure & $(n=1727)$ & $(n=796)$ & $P$ value \\
\hline All-cause mortality, risk (95\% Cl) & $5.7 \%(4.6 \%$ to $6.8 \%)$ & $6.8 \%(5.2 \%$ to $8.5 \%)$ & 0.23 \\
\hline $\mathrm{HF}$, risk $(95 \% \mathrm{Cl})$ & $10.5 \%(9.0 \%$ to $11.9 \%)$ & $9.4 \%(7.5 \%$ to $11.3 \%)$ & 0.37 \\
\hline VT/NF/arrest, risk (95\% Cl) & $1.6 \%(1.0 \%$ to $2.2 \%)$ & $3.3 \%(2.0 \%$ to $4.5 \%)$ & 0.018 \\
\hline ICD implantation, risk $(95 \% \mathrm{Cl})$ & $2.5 \%(1.7 \%$ to $3.3 \%)$ & $1.8 \%(0.9 \%$ to $2.7 \%)$ & 0.25 \\
\hline
\end{tabular}

The reported absolute risks of the outcomes for patients with acute myocarditis diagnosed during 2008-2018 versus during 2002-2007 standardised to the age, sex and comorbidity distribution of all patients were derived from multivariable Cox regression. The following covariates were included in the models: patient age, sex, hypertension, diabetes, chronic obstructive pulmonary disease, prior myocardial infarction, prior ischaemic heart disease, percutaneous cardiac intervention, coronary artery bypass grafting, stroke, peripheral artery disease, atrial fibrillation or flutter, HF, prior ventricular VT/VF/arrest and chronic kidney disease.

HF, heart failure; ICD, implantable cardioverter-defibrillator; VF, ventricular fibrillation; VT, ventricular tachycardia. 


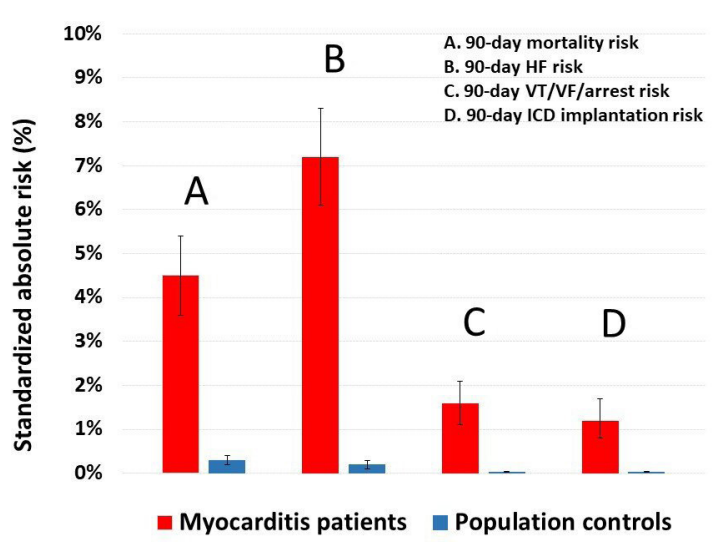

Figure 4 Standardised 90-day absolute risks of all-cause mortality, HF, ventricular arrhythmias and ICD implantation, with myocardial infarction or revascularisation events during the 90-day follow-up period as competing risks. The reported absolute risks for patients with acute myocarditis versus population controls standardised to the age, sex and comorbidity distribution of all patients were derived from multivariable Cox regression. The following covariates were included in the models: patient age, sex, hypertension, diabetes, chronic obstructive pulmonary disease, prior myocardial infarction, prior ischaemic heart disease, percutaneous cardiac intervention, coronary artery bypass grafting, stroke, peripheral artery disease, atrial fibrillation or flutter, HF, prior ventricular VT/VF/arrest and chronic kidney disease. HF, heart failure; ICD, implantable cardioverterdefibrillator; VF, ventricular fibrillation; VT, ventricular tachycardia.

CMR use was limited prior to 2007, but its subsequent use in diagnosing acute myocarditis increased substantially. CMR is relatively safe and accessible, and has very high sensitivity, specificity and accuracy in diagnosing acute myocarditis. ${ }^{27} 28$ Applying the three proposed criteria by Friedrich et al of T2-weighted focal myocardial oedema, T1-weighted early gadolinium enhancement suggesting hyperaemia and late gadolinium enhancement suggesting fibrosis or inflammation, identification of acute myocarditis by CMR is relatively accurate. ${ }^{28}$ However, there can be some technical limitations, and similar to EMB, timely performance is key for the accuracy in diagnosis. Interestingly, the recent focus on performing CMR in patients with MI and non-obstructive coronary artery disease also appears to have led to an increased detection of acute myocarditis. ${ }^{29}$ Furthermore, CMR could potentially be used for risk stratification into groups who are at risk of developing fatal cardiac complications, and those who are not. ${ }^{19-21}$ The studies in this field are limited, both in number of studies and of patients within the studies and should be a field of future research.

\section{Strengths and limitations}

Strengths of our study include the large sample size and the negligible loss to follow-up. The short-term risk assessment window increases the possibility of an underlying plausible and causal relation between the myocarditis condition and events. Limitations include the register-based, observational design and potential uncertainty regarding the myocarditis diagnosis, including the lack of results from the diagnostic work-up. Nevertheless, a diagnosis of acute myocarditis in the context of a CMR, a PET-CT or an EMB suggests high diagnostic certainty. The positive predictive value of acute myocarditis in the Danish National Patient Register was previously shown to be $>80 \%$ for patients $<60$ years of age, but $\approx 65 \%$ for older patients. ${ }^{18}$ Although event rates were lower for patients $<60$ years of age, this sensitivity analysis still found significantly higher mortality risk for patients with acute myocarditis relative to population controls, and as such, results were in agreement with the overall study results. In addition, the 90-day mortality risk was significantly elevated among patients with myocarditis who were examined with an EMB, indicating on one hand a more severe disease condition but also underscoring the likely causal association between myocarditis and fatal event risk. In continuation, staging of myocarditis including fulminant and non-fulminant conditions were otherwise not possible to define. Because myocardial ischaemia represents a common differential diagnosis of acute myocarditis, it was reassuring that results from analyses in which MI or revascularisation events during the 90-day follow-up period were considered competing risks were comparable to the overall results. Lastly, and as pointed out in the methods section, the study lacks several important clinical data and test results of troponins, ECGs (including QRS parameters), imaging modalities including echocardiography (including left ventricular ejection fraction), CMR, coronary artery status from CCTA and CAG and the actual EMB diagnosis and indication for ICD implantation. Nonetheless, the significantly elevated arrhythmic risk in the short run, which was consistent in younger patients with myocarditis, for whom the diagnostic performance of diagnosis coding has been shown to be of high quality, speaks towards a plausible link between myocarditis and an elevated arrhythmic risk. Future studies are needed to examine predictors of these adverse outcomes and the role and timing of cardiac monitoring to prevent these potentially fatal events. An important aspect of such future studies involves identification of the underlying causes for risk stratification and adverse outcome prevention.

\section{CONCLUSION}

This large nationwide register-based follow-up study of patients with acute myocarditis showed that patients with acute myocarditis have increased 90-day risks of all-cause mortality, HF, ventricular arrhythmias, cardiac arrest and ICD implantation when compared with age-matched and sexmatched background population controls. These findings 
suggest that patients with acute myocarditis may benefit from careful diagnostic work-up including cardiac monitoring in the early phase after diagnosis. Further studies focusing on risk stratification for these patients are warranted.

Contributors KHK, BT, AH and PS designed the study. KHK and FLL drafted the manuscript. KHK performed all statistical analyses. All authors provided substantial contributions to data interpretation and critical revisions, and all authors approved the final manuscript. KHK, FLL and BT are responsible for the overall content as guarantor and thereby accept full responsibility for the finished work and the conduct of the study, including access to the data.

Funding The authors have not declared a specific grant for this research from any funding agency in the public, commercial or not-for-profit sectors.

Competing interests KHK: Speaker's honoraria from Novartis. PS: Speaker for GE Healthcare, advisory board member (Novartis Pharmaceuticals Corp, AstraZeneca Pharmaceuticals, Biotronik), research grants Wics, Bayer and GE Healthcare. MP: Advisory board for AstraZeneca and Janssen-Cilag, speaker's free from AstraZeneca, Bayer, Boehringer Ingelheim and Janssen-Cilag. TZ: Speaker's fee from AstraZeneca. AH: Speaker for AstraZeneca, Cardiac Dimensions, DaiichiSankyo, GE Healthcare and Novartis.

Patient consent for publication Not applicable.

Provenance and peer review Not commissioned; externally peer reviewed.

Data availability statement Data may be obtained from a third party and are not publicly available. According to Statistics Denmark regulations, data used for this study cannot be shared, as data is stored on and may not leave secure servers on Statistics Denmark. Access to data on these servers can be granted upon adequate permission.

Open access This is an open access article distributed in accordance with the Creative Commons Attribution Non Commercial (CC BY-NC 4.0) license, which permits others to distribute, remix, adapt, build upon this work non-commercially, and license their derivative works on different terms, provided the original work is properly cited, appropriate credit is given, any changes made indicated, and the use is non-commercial. See: http://creativecommons.org/licenses/by-nc/4.0/.

\section{ORCID iDs}

Kristian Hay Kragholm http://orcid.org/0000-0001-9629-8670

Tomas Zaremba http://orcid.org/0000-0001-6365-6638

\section{REFERENCES}

1 Trachtenberg $\mathrm{BH}$, Hare JM. Inflammatory cardiomyopathic syndromes. Circ Res 2017;121:803-18.

2 Caforio ALP, Pankuweit S, Arbustini E, et al. Current state of knowledge on aetiology, diagnosis, management, and therapy of acute myocarditis: a position statement of the European Society of Cardiology Working Group on Myocardial and Pericardial Diseases. Europ Heart J Oxford University 2013;34:2636-48.

3 Gore I, Saphir O. Myocarditis; a classification of 1402 cases. Am Heart J 1947;34:827-30.

4 Harmon KG, Asif IM, Maleszewski JJ, et al. Incidence and etiology of sudden cardiac arrest and death in high school athletes in the United States. Mayo Clin Proc 2016;91:1493-502.

5 Basso C, Calabrese F, Corrado D, et al. Postmortem diagnosis in sudden cardiac death victims: macroscopic, microscopic and molecular findings. Cardiovasc Res 2001;50:290-300.

6 Te ALD WT-CC, Y-JJ L, Chen Y-YY, et al. Increased risk of ventricular tachycardia and cardiovascular death in patients with acute myocarditis during the long-term follow-up. Medicine 2017;0:e6633.

7 Grün S, Schumm J, Greulich S, et al. Long-term follow-up of biopsyproven viral myocarditis. J Am Coll Cardiol 2012;59:1604-15.
8 Chang J-J, Lin M-S, Chen T-H, et al. Heart failure and mortality of adult survivors from acute myocarditis requiring intensive care treatment - a nationwide cohort study. Int J Med Sci 2017;14:1241-50.

9 Lurz P, Eitel I, Adam J. Diagnostic performance of CMR imaging compared with EMB in patients with suspected acute myocarditis. JACC: Cardiovascular Imaging Elsevier 2012;5:513-24.

10 Gravanis MB, Sternby NH. Incidence of myocarditis. A 10-year autopsy study from Malmo, Sweden. Arch Pathol Lab Med 1991;115:390-2.

11 Kyto V, Saraste A, Voipio-Pulkki L-M, et al. Incidence of fatal myocarditis: a population-based study in Finland. Am J Epidemiol 2006;165:570-4.

12 Chen S, Hoss S, Zeniou V, et al. Electrocardiographic predictors of morbidity and mortality in patients with acute myocarditis: the importance of QRS-T angle. J Card Fail 2018;24:3-8.

13 Pedersen CB. The Danish civil registration system. Scand J Public Health 2011;39:22-5.

14 Lynge E, Sandegaard JL, Rebolj M. The Danish national patient register. Scand J Public Health 2011;39:30-3.

15 Kildemoes HW, Sørensen HT, Hallas J. The Danish national prescription registry. Scand J Public Health 2011;39:38-41.

16 Grant RL. Converting an odds ratio to a range of plausible relative risks for better communication of research findings. BMJ 2014;348:f7450.

17 Krogager ML, Torp-Pedersen C, Mortensen RN, et al. Shortterm mortality risk of serum potassium levels in hypertension: a retrospective analysis of nationwide registry data. Eur Heart $J$ 2017;38:104-12.

18 Sundbøll J, Adelborg K, Munch T, et al. Positive predictive value of cardiovascular diagnoses in the Danish national patient registry: a validation study. BMJ Open 2016;6:6.

19 R Core Team. R: a language and environment for statistical computing. $\mathrm{R}$ foundation for statistical computing, Vienna, Austria, 2017. Available: https://www.R-project.org/

20 D'Ambrosio A, Patti G, Manzoli A, et al. The fate of acute myocarditis between spontaneous improvement and evolution to dilated cardiomyopathy: a review. Heart 2001;85:499-504.

21 Aretz HT, Billingham ME, Edwards WD, et al. Myocarditis. A histopathologic definition and classification. Am J Cardiovasc Pathol 1987;1:1:3-14.

22 Caforio ALP, Calabrese F, Angelini A, et al. A prospective study of biopsy-proven myocarditis: prognostic relevance of clinical and aetiopathogenetic features at diagnosis. Eur Heart $J$ 2007;28:1326-33.

23 Ruiz-Ortiz M, Anguita-Sánchez M, Bonilla-Palomas JL, et al. Incidence and outcomes of hospital treated acute myocarditis from 2003 to 2015 in Spain. Eur J Clin Invest 2021;51:e13444.

24 Kodama M, Oda H, Okabe M, et al. Early and long-term mortality of the clinical subtypes of myocarditis. Jpn Circ J 2001;65:961-4.

25 Hauck AJ, Kearney DL, Edwards WD. Evaluation of postmortem endomyocardial biopsy specimens from 38 patients with lymphocytic myocarditis: implications for role of sampling error Mayo Clin Proc 1989;64:1235-45

26 Shanes JG, Ghali J, Billingham ME, et al. Interobserver variability in the pathologic interpretation of endomyocardial biopsy results. Circulation 1987;75:401-5.

27 Abdel-Aty H, Boyé P, Zagrosek A, et al. Diagnostic performance of cardiovascular magnetic resonance in patients with suspected acute myocarditis: comparison of different approaches. J Am Coll Cardiol 2005;45:1815-22.

28 Friedrich MG, Sechtem U, Schulz-Menger J, et al. International consensus group on cardiovascular magnetic resonance in myocarditis. cardiovascular magnetic resonance in myocarditis: a JACC white paper. J Am Coll Cardiol 2009;17:1475-87.

29 Heidecker B, Ruedi G, Baltensperger N, et al. Systematic use of cardiac magnetic resonance imaging in MINOCA led to a five-fold increase in the detection rate of myocarditis: a retrospective study. Swiss Med Wkly 2019;149:w20098. 